\title{
Better than riches
}

\author{
The involvement of online discussion sites in the identification of errors, anomalies and worse in \\ the published literature continues to demonstrate the usefulness of post-publication review. It also \\ highlights the ambiguous power of anonymity.
}

In early July both the French National Centre for Scientific Research (CNRS) and the Swiss Federal Institute of Technology Zurich (ETHZ) published the findings of independent investigations into allegations of misconduct against Professor Olivier Voinnet, a senior scientist with the CNRS who has been on secondment at ETHZ since November 2010. Both investigations found evidence of inappropriate figure manipulation and duplication stretching back over many years.

The CNRS was constrained by French law to only a brief statement summarizing their findings (http://go.nature.com/yLcXvw) while ETHZ released a 22-page report (http://go.nature.com/qw19NY) with only a few phrases redacted. The conclusion from both organizations was that figures published in a number of papers had been misrepresented or manipulated, although according to the CNRS "such inappropriate presentation of experimental data ... does not amount to fabrication". Similarly ETHZ took the fact that Voinnet was able to provide the original and raw data that "would have been sufficient to fully substantiate the authors conclusions" meant that this was "not a case of scientific misconduct as defined in ETH Zurich's Rules of Procedure". Rather than flawed research the problems resulted from the pressure to get data published, quickly leading to corners being cut and errors being made; in the deathless prose of ETHZ's press office: "Conducted properly - published incorrectly" (http://go.nature.com/eqESKX). Nevertheless, Voinnet's reputation is permanently tarnished.

Leaving aside the specifics of this case an interesting aspect is the channel through which the problems with these papers were initially communicated. In times gone by anyone who identified a problem with a scientific article would contact the journal in which it was published. The editors of Nature journals are very used to such complaints and investigate them carefully. We present them to the authors of papers asking for an explanation, and formal corrections or retractions are frequently published as a result. In the, thankfully, rare cases where we suspect that deliberate fraud may have taken place we will refer matters to a researcher's institution. Throughout we protect the identity of the person who lodged the concern. It may be that this happened with Voinnet's papers, but it was the very public voicing of doubts on the website PubPeer that triggered the institutional investigations.

PubPeer was established as a response to the paucity of discussion or 'postpublication peer review' taking place on journal websites, despite most providing the opportunity for readers to engage in online commenting. It provides a centralized forum in contrast to the multiple systems of different publishers. More importantly perhaps, PubPeer encourages anonymity.

There are strong arguments for the reviewing and discussion of research to be anonymous. To focus attention on what is being said and not who is saying it. To protect the commenter, allowing them to express robust and honest opinions without fear of reprisals from those they are criticizing. This is perceived as such a problem that the creators of PubPeer, "a diverse team of early-stage scientists in collaboration with programmers" themselves remain anonymous as they feel that even facilitating this kind of discussion could anger colleagues. Commenting does not have to be anonymous but aside from the authors of papers responding to points and questions, most entries on PubPeer are. Comments come either from individuals with PubPeer accounts, available to any published researcher with an academic or research institute e-mail address (I have an account myself), or from non-account holders in which case they are moderated before being displayed.

PubPeer's aim of promoting open discussion of published science is admirable but anyone with experience of online forums, in whatever sphere, will not be surprised that posts tend towards negative criticism, rather than forward looking debate, kicked off by the identification of a potential error or inaccuracy in the content of a paper. PubPeer has had considerable success in identifying potential problems in papers, but there is a danger of the site developing a triumphalist tone with contributors revelling in catching-out researchers in even minor errors. The ETHZ report goes so far as to say that "the potential exists to exploit [PubPeer] in cases of scientific conflict" and urges the scientific community to "open a debate about how it wants to deal with suspicions of misconduct, and how it wants to protect itself from perversion of all sorts".

Anonymity is a powerful feature of the practice of science. It attempts to remove personality from the processes but in so doing can discard relevant contexts. Traditionally referees of scientific papers are anonymous (to authors, though not to editors) but it is frequently suggested that the authorship of a paper should also be unknown while it is being assessed. And if the authorship of a paper should be undisclosed to referees why not to editors too? The mechanics of achieving this may be difficult but some form of 'tripleblind' review should be possible. Indeed the editors of Molecular Cell have taken a step in that direction, making a firstpass assessment of papers based on titles and abstracts only, before drilling down more deeply into the specifics of the paper (http://go.nature.com/cqkDcw). Whether that is affecting what Molecular Cell reviews and publishes is as yet unknown.

Like the other Nature research journals, Nature Plants offers blind review but the number of authors taking-up the option is very low. We cannot say with any certainty why this should be, despite blind review so often being raised as a way to remove biases from peer review. Perhaps no author thinks that having their own name attached to a paper will decrease its likelihood of receiving a favourable assessment. Perhaps they feel that the act of choosing anonymity suggests they have something to hide. Or perhaps it is pride; pride in themselves and the institution they come from, that makes them reluctant to be unknown. As one researcher said to me "I still cannot understand why anyone would do such a thing".

A scientist's name is a valuable commodity. There is power in its masking as well as its disclosure. It carries with it the weight of a reseacher's past achievements and yet is so fragile that it should never be risked for short-term gains. 